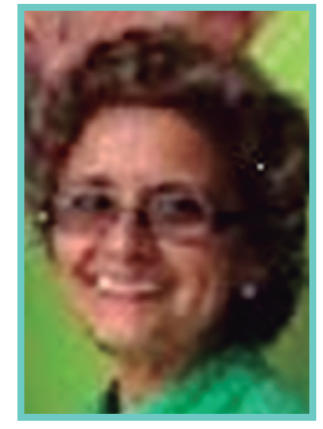

María del Rosario

Rivas Plata Álvarez

\title{
PRÁCTICAS LECTORAS TRADICIONALES DE LOS ACOMPAÑANTES PEDAGÓGICOS
}

\section{RESUMEN}

La presente investigación profundizó en el tema de la tendencia y motivación hacia la lectura de los acompañantes pedagógicos del programa presupuestal con enfoque por resultados "Logros de Aprendizaje de los estudiantes de Educación Básica regular" - PELA.

\section{PALABRAS CLAVE}

Lectura, motivación hacia la lectura, motivación y actividad lectora.

\section{ABSTRACT}

This research delved into the theme of the trend and motivation for reading of the budget accompanying educational program focusing on results "Learning achievements of students in regular basic education" PELA.

\section{KEYWORDS}

Reading, reading motivation, motivation and reading activity.
T a lectura es un proceso que se inicia desde que se está pequeño, es más, antes de leer de manera formal. Se va consolidando para hacerse más completo durante toda la vida. Es la práctica, el ejercicio continuo que hace ser más competente y adquirir habilidad, pero también es el factor tiempo que ayuda a madurar este proceso e ir consolidándolo y fortaleciéndolo si se mantiene en continua práctica.

Es así que al llegar a espacios académicos, se espera contar con habilidades lectoras desarrolladas. Quiere decir que la historia de vida de un lector proporciona datos valiosos para entender las características de los lectores y de los no lectores.

En el caso de los maestros de carrera, la necesidad que desarrollen hábitos de lectura y escritura de manera autónoma, es un pendiente que se debe resolver hoy en día, dado que son ellos los que tienen un rol protagónico en el proceso, porque son los encargados desde la escuela, de crear en los estudiantes 
estos hábitos, además porque el docente sirve de modelo y referente para los estudiantes, quien al ser lector y escritor provoca en sus estudiantes dinamizar en la escuela estos procesos.

Respecto de este tema, existe socialmente diversos "mitos". Uno de ellos y el más popularizado es que si una persona ejerce la carrera de maestro/a, indiscutiblemente posee la habilidad para leer y escribir de manera correcta.

En la investigación sobre el efecto del programa PRONAFCAP especialización en el desarrollo de habilidades comunicativas en las docentes del nivel de Educación Inicial de Lima Metropolitana (Rivas Plata, M., 2014), la hipótesis sostenía que las docentes del nivel de Educación Inicial al participar de los estudios de segunda especialidad desarrollando las áreas curriculares de matemática y comunicación, elevarían su nivel de comprensión lectora. Los resultados arrojaron que al finalizar el programa las maestras alcanzaron el puntaje más alto en la dimensión de Comprensión literal únicamente, en los otros niveles (reorganización de la información, comprensión mediante inferencias, lectura crítica y apreciación lectora) más elevados de comprensión el avance fue de un $20 \%$.

Quiere decir, que el nivel más alto de comprensión que tiene que ver con que el lector cuente con la capacidad para hacerse un juicio sobre la totalidad del texto, a partir de la reflexión del mismo, sea capaz de criticar el documento leído de manera objetiva y valore su lectura (Monson, D., 1989), las maestras del nivel de Educación Inicial no lograron alcanzar el nivel esperado.

Solo llegaron a desarrollar de manera primaria su nivel de comprensión, el que está directamente relacionado con dos habilidades que intervienen en la comprensión literal: reconocer y recordar. El nivel más elevado en este proceso de comprensión literal tiene que ver con el recordar, ya que el lector recupera información de la memoria sin ninguna pista o ayuda, a diferencia del proceso de reconocer, donde sí necesita alguna pista o estímulo visible para traer a su memoria lo leído. En la prueba de entrada de comprensión alcanzaron el mismo nivel (de 2.78 en la entrada a 2.86 a la salida).

Sin embargo, esta realidad no es ajena a los docentes del nivel de Educación Primaria, ya que el diagnóstico que se levantó sobre las prácticas lectoras de los acompañantes pedagógicos, muestran cifras que indican las mismas dificultades de un docente de Educación Inicial con los de un docente de Educación Primaria en relación con sus hábitos de lectura, los cuales están muy básicamente desarrollados, o no los tienen.

Hay estudios sobre docentes que muestran la debilidad de los maestros y maestras, donde el perfil de lector no es el más adecuado para un profesional cuya labor está directamente relacionada con el desarrollo de habilidades de lectura y escritura en los niños. Esta preocupación fue la que motivó indagar sobre las prácticas lectoras de los docentes. En esta oportunidad trabajando con los Acompañantes Pedagógicos.

Pertenece al programa presupuestal con enfoque por resultados "Logros de Aprendizaje de los estudiantes de Educación Básica Regular” - PELA.

El acompañante pedagógico es el responsable de visitar periódicamente a los docentes de las escuelas públicas focalizadas, donde los niños y maestros han obtenido bajos resultados en su rendimiento. Da soporte pedagógico al maestro de las instituciones educativas multigrado a través de tres formas de intervención: visita en aula, micro-taller y talleres regionales. En las tres estrategias, brinda asesoría al maestro con la finalidad de revertir la situación inicial encontrada.

\section{2 unı}


Fueron seleccionados de acuerdo a su región y son los mejores profesionales con los que cuenta cada zona, de tal manera que se espera que al ser ellos buenos maestros cuando ejercieron su rol, igualmente puedan ayudar al maestro de escuela ahora para mejorar sus prácticas pedagógicas.

Se levantó la información para comprender el comportamiento lector en el momento actual que los acompañantes pedagógicos están estudiando una segunda especialidad y que muestran debilidad en su rendimiento académico, de tal manera que se cuente con aspectos concretos sobre su práctica de lectura, además de identificar las motivaciones, lugares y vías de acceso a los libros y uso del tiempo libre dedicado a la lectura. El estudio se ha dirigido a un grupo humano poco abordado por la investigación: adultos docentes que cursan estudios de segunda especialidad.

Para ello se aplicó un cuestionario de 51 preguntas a 350 acompañantes pedagógicos, que luego fueron más profundizadas por medio de grupos focales.

Del total de acompañantes pedagógicos, $46 \%$ corresponden a acompañantes de sexo masculino y $54 \%$ de sexo femenino.

Son docentes del nivel de Educación Inicial un 20\%, y 80\% docentes del nivel de Educación Primaria.

Un 43\% son licenciados en educación inicial, primaria o secundaria, y el 57\% son titulados en educación egresados de un ISP (cifra que incluye un 2\% de docentes que han logrado el bachiller en educación).

Respecto de la edad, la mayor concentración se encuentra en el rango de edad de 35 a 56 años (91\%). Muy por debajo un 5\% pertenecen a los acompañantes que se encuentran entre los 34 a 24 años, y por último con un 3\% los acompañantes mayores de 57 hasta 67 años.
El diagnóstico estuvo enmarcado en el análisis del comportamiento lector en tres prácticas relacionadas con la lectura.

Motivación ante la lectura:

- Vías de acceso para la lectura.

- Uso del tiempo libre dedicado a la lectura.

\section{¿QUE LEEN LOS ACOMPAÑANTES PEDAGÓGICOS? SU MOTIVACIÓN E IDENTIDAD LECTORA}

Considerar la historia lectora del grupo, fue la finalidad de levantar información al respecto. Por ello, se consultó a los maestros si recordaban un libro en especial que leyeron y las razones que motivaron su lectura.

El $70.5 \%$ de los acompañantes pedagógicos recordaron haber leído textos literarios. Entre los textos más leídos se encuentran las novelas, cuentos, y en general textos literarios de la literatura peruana. A continuación y con un $14.8 \%$ novelas de aventura de autores extranjeros; por último, se encuentran los textos técnicos, que generalmente han sido ubicados en esta categoría los diccionarios de filosofía, con un $10.5 \%$.

Recuerdan los textos leídos porque la trama de la historia y contenido están relacionados con aspectos sociales y con la realidad peruana, por lo tanto los contenidos de las lecturas coinciden con la vida de ellos o de sus familias, o los hace recordar momentos de su infancia. Sin embargo, un porcentaje importante manifiesta que recuerda la lectura por los sentimientos de tristeza y pasión que contenía la obra.

Indicaron, igualmente, que los espacios de lectura se dieron antes de ejercer su rol de acompañantes pedagógicos, y que ahora no cuentan con dichos espacios. 
¿QUÉ LES OFRECE LA COMUNIDAD A LOS ACOMPAÑANTES PEDAGÓGICOS PARA CONTRIBUIR CON SU FORMACIÓN?

La formación permanente del acompañante pedagógico es una tarea que le pertenece de manera compartida al propio acompañante y a su región. El rol que ejercen está directamente relacionado con mejorar las prácticas de los docentes que se encuentran en escuelas focalizadas y que poseen bajo rendimiento. Son entonces los acompañantes pedagógicos uno de los mediadores para propiciar el mejor rendimiento del docente y por ende de los niños.

Para lograr los objetivos que persigue la educación en la región, necesita contar con cuadros de profesionales preparados y actualizados con las últimas innovaciones que se dan en los espacios educativos.

Requiere de un componente clave (Tovar, R., 2008), su formación como lector y como escritor. Sin el desarrollo de ambos aspectos, no podría pensarse en un acompañante pedagógico preparado para ejercer su rol.

Frente a la consulta sobre la última lectura realizada y las motivaciones para su lectura, el $99 \%$ dio como referencias a los módulos repartidos durante el programa de segunda especialidad, las separatas de lecturas complementarias proporcionadas y otra literatura especialidad en referencia con los bloques temáticos desarrollados en el programa. Las motivaciones frente a la lectura estuvieron directamente relacionadas con su proceso de formación; es decir, que las prácticas de lectura se deben a motivaciones instrumentales y que lo hacen dada la necesidad de cumplir con sus estudios.

Cuando se indagó sobre las razones por las cuales no han tenido más prácticas de lectura, señalaron el poco tiempo que tienen para leer, así como las limitaciones para adquirir libros, ya sea por los altos costos o porque las zonas donde viven no cuentan con librerías especializadas de textos más técnicos para su profesión, incluso señalaron que las zonas no cuentan con bibliotecas; por lo tanto, las prácticas asociadas con la lectura que se logran por medio de la concurrencia a la biblioteca no existen en las zonas.

Al consultar sobre la posibilidad de hacer uso de otras vías de acceso a la lectura, como el uso del Internet y las redes sociales, indicaron las dificultades que presenta la conectividad de Internet en su zona que no posee banda ancha, lo que hace muy lento el servicio.

Sanjuán, M. (2011) señala que el proceso lector es un hecho cultural e histórico que se basa en la relación que se da entre la literatura y el lector. Además, acompaña el proceso el componente emocional, la experiencia individual de la lectura, la posibilidad de que la literatura transforme al lector.

Esta idea indica que es la cultura, la comunidad, la responsable de dotar de condiciones para que el profesional de la educación cuente con la posibilidad de seguir formándose y perfeccionándose; sin embargo, el espacio cultural de las regiones no están preparados hasta hoy para invertir en dotar a su comunidad de espacios que les permitan contar con literatura al alcance de los profesionales. $\mathrm{Ni}$ que decir de los estudiantes de educación básica que solo cuentan con los libros de texto proporcionados por el Estado en las zonas rurales del país. Por lo tanto, los conocimientos no se renuevan, y lo que es más delicado aún, la necesidad de que los docentes sean lectores para que puedan promover estos procesos en sus pares no se da. Desde esta visión compleja que viven las regiones, los profesionales y los estudiantes no tendrán un mejor futuro, ni tampoco sus regiones. 
USO DEL TIEMPO LIBRE DEDICADO A LA LECTURA

Se reconoce el valor que tiene la lectura para conformar sociedades más perfectas; sin embargo, el acceso a la lectura es cada vez más restringido, dado que el gusto por ella va disminuyendo. Entonces y tal como lo afirman Yubero, S.; Larrañaga, E.; Torremocha, P., (2004), la diferencia ya no está en saber o no saber leer, sino en tener hábitos de lectura o no ser lector.

Para indagar acerca de lo que hacen los acompañantes pedagógicos en su tiempo libre, se formularon preguntas relacionadas con sus gustos a futuro, las actividades relacionadas con el descanso que tienen en el momento que no trabajan y como invierten su tiempo libre.

La información se recogió en dieciocho opciones que consideraban actividades diversas (deportivas, familiares, culturales, académicas) $y$, entre ellas, actividades de lectura y escritura.

El acompañante pedagógico debía de asignarle un puntaje de 1 al 18 a cada opción, donde 1 era la opción que tenía mayor prioridad y 18 la de última prioridad.

Los resultados arrojaron que el mayor porcentaje se concentra en las opciones 1 al 5 y 16 al 18; es decir, los acompañantes pedagógicos invierte su tiempo libre dedicándolo a la familia, no haciendo "nada" y escribiendo.

\section{A MODO DE CONCLUSIONES}

Si bien es cierto que la información recogida sigue en construcción, los resultados iniciales analizados en el apartado anterior permiten extraer las siguientes conclusiones:

- Los acompañantes pedagógicos manifiestan una débil tendencia hacia la lectura, producto de su historia como lectores.
- La motivación ante la lectura que manifiestan los acompañantes pedagógicos está directamente relacionada con su quehacer formativo en este momento; por lo tanto, más que una motivación sienten la presión de hacerlo.

- Los acompañantes pedagógicos que ejercen su labor en las zonas rurales, no cuentan con condiciones básicas para mejorar su formación académica, dado que la comunidad no les asegura vías de acceso para la lectura.

- Los acompañantes pedagógicos desarrollan un trabajo en extremo operativo, además de invertir mucho tiempo en el desplazamiento a las zonas donde se ubican las escuelas y los maestros. Este hecho hace que se encuentren cansados, y al contar con espacios libres, no los dedican necesariamente a la lectura.

- Algunas características de la intervención del programa de segunda especialidad tendrían que adecuarse a las condiciones del adulto estudiante universitario que realiza un trabajo en zonas alejadas.

- Se hace necesario revisar los enfoques con los que se conciben los programas de segunda especialidad para docentes en servicio, en especial para los acompañantes del Pela.

- El estado está invirtiendo permanentemente en mejorar la calidad educativa de los niños y niñas, sin advertir que son los docentes quienes presentan dificultades para acceder de manera comprensiva a espacios de lectura y escritura.

- Los programas dedicados a los maestros en servicio necesariamente deben incluir una etapa "cero" de formación, para ejercitar las habilidades lectoras, de tal manera que los docentes estudiantes muestren mejores resultados durante su periodo de formación en servicio. 


\section{REFERENCIAS}

Monson, Dianne L. (1989). Rastreando en un libro y más allá de él: las reacciones ante la literatura. Madrid: Visor.

Rivas Plata, M. (2014). Efectos del programa PRONAFCAP especialización en el desarrollo de habilidades comunicativas en las docentes de educación inicial de Lima Metropolitana. (Tesis de Maestría). Lima, Perú: Universidad Ricardo Palma.

Sanjuán Álvarez, Marta (2011). De la experiencia de la lectura a la educación literaria. Análisis de los componentes emocionales de la lectura literaria en la infancia y la adolescencia. Ocnos, 7, 85-100.
Disponible en: file:///C:/Users/user/Downloads/212-940-1PB.pdf

Tovar, Rosa María. La formación como lector y escritor: un reto para el docente. Educere [en línea] 2009, 13 (Enero-marzo). [Fecha de consulta: 8 de septiembre de 2015], disponible en: http:// www.redalyc.org/articulo.oa?id=35614571013

Yubero, S.; Larrañaga, E.; Torremocha, P. (2004). Valores y lectura. Estudios multidisciplinarios. Ediciones de la Universidad de Castilla - La Mancha. 